\title{
Synergistic neuroprotective effects of Geniposide and ursodeoxycholic acid in hypoxia-reoxygenation injury in SH-SY5Y cells
}

\author{
FAFENG CHENG $^{1 *}$, CHONGYANG MA $^{1 *}$, LIANGMING SUN ${ }^{2}$, XIAOYU ZHANG ${ }^{1}$, \\ CHANGMING ZHAI $^{3}$, CHANGXIANG LI ${ }^{1}$, SHUANG ZHANG ${ }^{1}$, BEIDA REN ${ }^{1}$, \\ SHULING LIU $^{1}$, SONGNAN LIU ${ }^{1}$, XIANGJUN YIN ${ }^{1}$, XUEQIAN WANG ${ }^{1}$ and QINGGUO WANG $^{1}$ \\ ${ }^{1}$ School of Basic Medical Sciences, Beijing University of Chinese Medicine, School of Basic Medical Sciences, \\ Chao Yang, Beijing 100029; ${ }^{2}$ Guang'anmen Hospital, China Academy of Chinese Medical Sciences, Beijing 100053; \\ ${ }^{3}$ Beijing University of Chinese Medicine Third Affiliated Hospital, Beijing 100029, P.R. China
}

Received June 15, 2017; Accepted October 26, 2017

DOI: 10.3892/etm.2017.5395

\begin{abstract}
Endoplasmic reticulum stress (ERS) and autophagy activation play important roles in the process of cerebral ischemia/reperfusion (I/R) injury. The synergistic protective effects of Geniposide and ursodeoxycholic acid against cellular apoptosis caused by oxygen-glucose deprivation-reoxygenation (OGD/R) were investigated using a Cell Counting Kit-8 assay, lactate dehydrogenase (LDH) assay, flow cytometry, quantitative polymerase chain reaction (qPCR), and western blotting to examine cellular viability, apoptosis, reactive oxygen species (ROS) levels, mRNA and protein levels, respectively, in relation to ERS and autophagy. We found that pretreatment with Geniposide improved cellular viability. Moreover, treatment with a combination of Geniposide and Tauroursodeoxycholic acid (TUDCA) (GT) protected injured cells better than Geniposide alone. Further studies showed that the increase in cellular ROS levels, and the overexpression of mRNA and proteins related to OGD/R-induced ERS and autophagy, were both counteracted by GT. Our study indicates that the protective effects of GT on OGD/R-induced apoptosis in SH-SY5Y cells are associated with the inhibition of ERS and autophagy.
\end{abstract}

Correspondence to: Dr Xueqian Wang or Qingguo Wang, School of Basic Medical Sciences, Beijing University of Chinese Medicine, School of Basic Medical Sciences, 11 Beisanhuandong Road, Chaoyang, Beijing 100029, P.R. China

E-mail: shirlyding@163.com

E-mail: wangqg8558@163.com

*Contributed equally

Key words: geniposide, ursodeoxycholic acid, oxygen-glucose deprivation, endoplasmic reticulum stress, autophagy, apoptosis

\section{Introduction}

Cerebral ischemia is a life-threatening disease with high morbidity and mortality rates worldwide. As the pathogenesis of stroke is very complex, there are currently no clinically effective therapies, making the need to develop new therapies ever more urgent. Endoplasmic reticulum stress (ERS) and autophagy activation play important roles in cerebral ischemia/reperfusion (I/R) injury, which is a double-edged sword as their activation will affect the fate of neurons following $I / R$ injury (1). These mechanisms both help to clean up damaged organelles and promote energy and molecule recycling. However, their activation can also induce cell death, for example by apoptosis, which is a key mechanism that leads to cell death following cerebral ischemia (2). Several lines of evidence suggest that there is communication between ER stress and autophagy (3). They are regarded as new therapeutic targets for ischemic stroke and other central nervous system diseases.

The neuroprotective effects of Jingzhi Qingkailing (JZQKL) injection, which consists of cholic acid, hyodeoxycholic acid, baicalin, and jasminoidin, and was developed from a famous anti-cerebral ischemia Chinese medicine, Qingkailing (QKL), are well established (4). Our previous studies have highlighted the neuroprotective effects of Geniposide and cholic acid compositions, especially Tauroursodeoxycholic acid (TUDCA) (5). Geniposide and TUDCA are pharmacologically active compounds purified from the two Chinese herbs Gardenia jasminoides Ellis and Bezoar, respectively, which have been used for the treatment of stroke for thousands of years $(6,7)$. Recent data suggest that Geniposide may be induce a wide range of biological processes, and thus likely exerts its neuroprotective effects through a variety of mechanisms. For example, Geniposide has been shown to protect neurons from oxidative damage by increasing the expression of anti-apoptotic proteins, including Bcl-2 and heme oxygenase- 1 (HO-1) (8). Geniposide has also been demonstrated to increase adenosine triphosphate (ATP) generation, mitochondrial membrane potential (MMP), cytochrome c oxidase $(\mathrm{CcO})$ 
and caspase-3 and -9 activity, reduce reactive oxygen species (ROS) production and cytochrome c leakage, as well as inhibit apoptosis (9). Finally, some studies have shown that Geniposide exerts its neuroprotective effects in vivo by inhibiting inflammation and ameliorating amyloid pathology (10). Moreover, it has been shown to improve cognition (10). TUDCA, an endogenous bile acid, is formed by the conjugation of ursodeoxycholic acid (UDCA) with taurine. TUDCA has been shown to have neuroprotective effects in a variety of experimental systems, including models of neurodegenerative disorders such as Alzheimer's disease and Huntington's disease (11-13). In addition, it has been shown to protect against damage induced by ischemia and hemorrhagic stroke $(12,14)$. The molecular mechanisms underlying the neuroprotective effects of TUDCA appear to be complex and may engage a number of different molecular targets, possibly involved in gene regulation, resulting in robust anti-apoptotic, anti-inflammatory, immunomodulatory, and antioxidant effects $(15,16)$.

In the clinical practice of Traditional Chinese Medicine (TCM), Gardenia jasminoides Ellis and Bezoar are always used together in one formula, such as in Niuhuang Shangqing or Angong Niuhuang pills, to treat neurovascular diseases $(17,18)$. The neuroprotective effects of Geniposide have been well established, and our previous studies have shown that the effective components of Bezoar alleviate cerebral ischemic injury by inhibiting ERS (5). Moreover, TUDCA has been shown to be more effective than taurine and UDCA both in vivo and in vitro (19). However, the synergistic effects of the two components combined remains to be fully elucidated. In the present study, we assessed the cytoprotective potential of Geniposide alone or in combination with TUDCA against OGD/R-induced injury to SH-SY5Y cells.

\section{Materials and methods}

Cell culture. SH-SY5Y cells were were obtained Shanghai Institute of Biochemistry and Cell Biology (Shanghai, China), cultured in Dulbecco's Modified Eagle's Medium (DMEM) containing $10 \%$ (v/v) fetal bovine serum (Invitrogen, Carlsbad, CA, USA), and maintained in $5 \%(\mathrm{v} / \mathrm{v}) \mathrm{CO}_{2} / 90 \%$ (v/v) humidity at $37^{\circ} \mathrm{C}$. Cells were plated onto 96 -well culture plates at a density of $1 \times 10^{4}$ cells/well for the cytotoxicity assays, or $60-\mathrm{mm}$ culture dishes at a density of $1 \times 10^{6}$ cells/well for western blot, qPCR and flow cytometry. After $24 \mathrm{~h}$, the culture medium was replaced with serum-free DMEM supplemented with $0.12 \mathrm{mM}$ formaldehyde, and different concentrations of drugs were used to treat the cells as indicated in the results section.

Oxygen-glucose deprivation and reoxygenation procedure. OGD/R was performed with the AnaeroPack system, which includes a rectangular container $(9.5 \times 6.75 \times 3.25 \mathrm{in} ; 2.5 \mathrm{l})$ and one AnaeroPack sachet. Briefly, SH-SY5Y cells were plated in DMEM. After treatment, the cells were incubated in the sachet with a BBL disposable anaerobic indicator strip and placed into the container. After $60 \mathrm{~min}$ of incubation, the oxygen concentration was less than $1 \%$ and the $\mathrm{CO}_{2}$ concentration was approximately $18 \%$. After $12 \mathrm{~h}$, the cells were incubated for $2 \mathrm{~h}$ for reoxygenation and glucose restoration. In the normoxia control group, the cells were cultured with DMEM containing glucose under normal conditions.
Cell viability assessment. The viability of SH-SY5Y cells was determined using a CCK-8 cell viability test Kit (CK04; Dojindo Laboratories, Kumamoto, Japan). Briefly, $10 \mu 1$ per well CCK-8 solution was added to the culture medium. The absorbance value (A) was measured at $450 \mathrm{~nm}$ using a spectrophotometer (Multiskan FC; Thermo Fisher Scientific, Waltham, MA, USA). The percentage of viable cells was calculated using the following formula: cell viability $(\%)=(\mathrm{A}$ of experiment well/A of control well) x $100 \%$.

LDH assay. SH-SY5Y cells were cultured in a 96-well culture plate at a density of $4.0 \times 10^{3}$ cells $/ \mathrm{cm}^{2}$ for $24 \mathrm{~h}$. Each treatment group consisted of a set of six wells. The medium was removed from each well post-treatment. The media samples were centrifuged at 1,000 rpm for $1 \mathrm{~min}$, and the supernatants were collected and subsequently used in the LDH assay, which was performed according to the manufacturer's instructions.

Quantitative PCR analysis. Total RNA was extracted from cells lysed using TRizol reagent (Takara Bio, Dalian, China) per the manufacturer's instructions (19). The quality and quantity of the RNA purity were assessed using a spectrophotometer and standard electrophoresis. cDNA was synthesized from $1 \mu \mathrm{g}$ RNA and reverse transcribed using a PrimerScript ${ }^{\mathrm{TM}}$ RT reagent kit (Takara Bio). The mRNA expression levels were quantified using a TaqMan ${ }^{\mathrm{TM}}$ Assay kit (Applied Biosystems Life Technologies, Foster City, CA, USA) in accordance with the manufacturer's instructions. The primer sequences used for CHOP were as follows: 5'-CACTCTTGACCTGCTTC-3' (forward) and 5'-AGTCGCCTCTACTTCCCT-3' (reverse). The product size was approximated at 307 base pairs (bp). The primers for beclin-1 were as follows: 5'-CGTGGAGAAAGG CAAGATT-3' (forward) and 5'-AGAACTGTGAGGACACCC AAG-3' (reverse). The product size was approximated at $152 \mathrm{bp}$. The primers for glyceraldehyde-3-phosphatedehydrogenase (GAPDH), the internal control, were as follows: 5'-CCATGG AGAAGGCTGGGG-3' (forward) and 5'-GTCATCCATGAC AACTTTG-3' (reverse). The product size was approximated at $195 \mathrm{bp}$. Each reaction was performed in triplicate using an ABI 7500 fast RT- PCR system. Gene expression levels were calculated based on the threshold cycle $(\mathrm{Cq})$ value. The relative mRNA expression levels in the samples were assessed using the comparative delta-delta $\mathrm{Cq}$ method (TaqMan Relative Quantification Assay software), adjusted to the mRNA expression level of GAPDH.

Flow cytometry analysis. Flow cytometry was used to assess the percentage of apoptotic cells in the different treatment groups. The cells were stained using an Annexin V-FITC/PI double staining kit (AD10; Dojindo Laboratories) per the manufacturer's instructions.

Detection of ROSusing the dichloro-dihydro-fluorescein diacetate (DCFH-DA) assay and flow cytometry. SH-SY5Y cells were seeded into 6 -well culture plates $\left(4.0 \times 10^{3}\right.$ cells $\left./ \mathrm{cm}^{2}\right)$ and cultured for $24 \mathrm{~h}$. The medium was removed from the wells after treatment. The cells were rinsed once with $0.1 \mathrm{M}$ phosphate buffered saline (PBS) and passaged using $0.25 \%$ trypsinization for $2 \mathrm{~min}$. Cells were collected in centrifuge tubes and centrifuged at 1,500 rpm for $5 \mathrm{~min}$, and the 
supernatants were discarded. DCFH-DA $(200 \mu \mathrm{l})$ was added to each tube for $20 \mathrm{~min}$ at $37^{\circ} \mathrm{C}$ in the absence of light. A keratinocyte serum-free medium $(500 \mu \mathrm{l})$ was added to rinse the cells, and cells were centrifuged at 1,000 rpm for $5 \mathrm{~min}$. The supernatants were discarded, and $200 \mu 1$ keratinocyte serum-free medium were added to the cells for testing.

Western blot analysis. Total protein was extracted using a kit (KGP250; Nanjing KeyGen Biotech Co., Ltd., Nanjing, China). Whole cell lysates were separated by $10-15 \%$ sodium dodecyl sulfate polyacrylamide gel electrophoresis (SDS-PAGE), and then transferred to a nitrocellulose membrane. The membrane was blocked with 5\% skimmed milk powder in Tris-buffered saline-Tween-20 (TBS-T; 0.1\% Tween-20 in TBS) for $1 \mathrm{~h}$ at room temperature and incubated overnight at $4^{\circ} \mathrm{C}$ with antibodies against Beclin-1 (1:1,000, ab62557; Abcam, Cambridge, UK), CHOP (1:1,000, ab11419; Abcam), and $\beta$-actin (1:2,000; Santa Cruz Biotechnology, Inc., Santa Cruz, CA) followed by incubation with horseradish peroxidase-conjugated goat anti-rabbit IgG antibody (1:3,000; Proteintech Group Inc., Hubei, China). Immunoreactive bands were visualized using a chemiluminescence kit (ECL kit; Santa Cruz Biotechnology, Inc.), and protein bands were scanned using Chemi Imager 5500 V2.03 software. The integrated density value (IDV) for each band was calculated with a computer-aided image analysis system (Fluor Chen 2.0).

Statistical analysis. All data were expressed as means \pm standard deviation (SD). Data were analyzed using a one-way analysis of variance (ANOVA) followed by a Bonferroni correction for multiple comparisons. $\mathrm{P}<0.05$ was considered to indicate a statistically significant difference.

\section{Results}

The synergistic effects of geniposide and TUDCA on SH-SY5Y cell viability. To investigate the synergistic protective effects of Geniposide and TUDCA against OGD/R (oxygen glucose deprivation-reoxygenation)-induced cell death, a Cell Count Kit-8 (CCK-8) assay was used to assess cellular viability in SH-SY5Y cells. We observed a concentration-dependent reduction in OGD/R-induced injury with increasing concentrations of Geniposide (Fig. 1A). A neuroprotective effect was observed following treatment with TUDCA at a concentration of $25 \mu \mathrm{g} / \mathrm{ml}$ only $(\mathrm{P}<0.05$; Fig. 1B). Treatment with a combination of Geniposide and TUDCA (GT) at a constant 20:1 Geniposide:TUDCA ratio increased the proliferation of SH-SY5Y cells $(\mathrm{P}<0.001)$ more than Geniposide alone following OGD/R treatment (Fig. 1C). Therefore, these concentrations were used in further experiments in combination. However, no statistically significant decreases in lactate dehydrogenase (LDH) activity were observed with these agents when compared to cells treated with OGD/R alone (data not shown).

Synergistic anti-apoptotic effects of geniposide and TUDCA. To investigate the synergistic effects of combined treatment with GT on OGD/R-induced SH-SY5Y cell injury, we examined apoptosis in SH-SY5Y cells treated with Annexin V-FITC/PI using flow cytometry (Fig. 2). As expected, the Annexin V/PI flow cytometric apoptosis assay
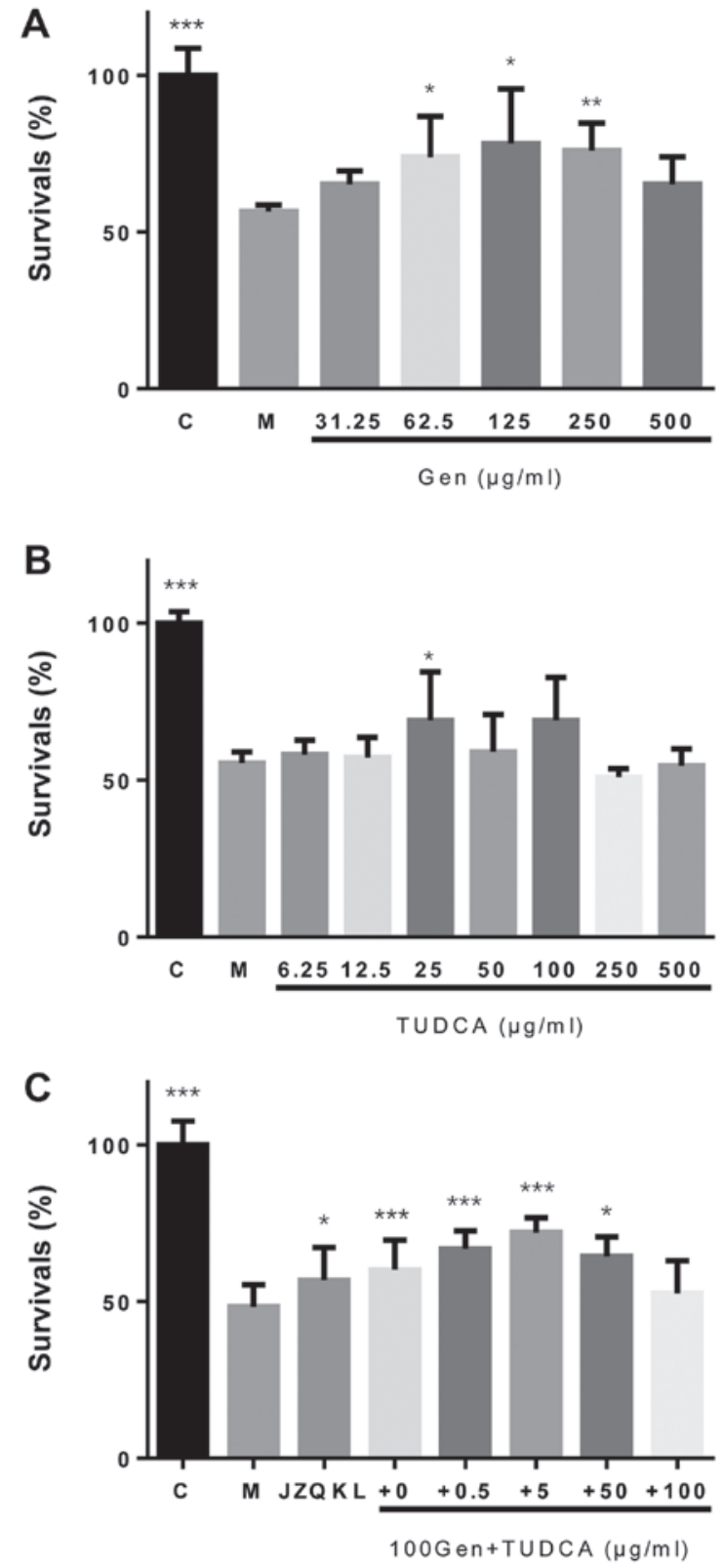

Figure 1. CCK8 assay for cellular viability. (A) Neuroprotective effects of Geniposide in SH-SY5Y cells exposed to OGD/R. The cells in the model group $(\mathrm{M})$ and those in the different treated groups were subjected to the OGD/R procedure. In the control group (C), the cells were cultured under normal conditions. (B) TUDCA does not exert neuroprotective effects in SH-SY5Y cells exposed to OGD/R. (C) Synergistic effects of Geniposide and TUDCA in SH-SY5Y cells exposed to OGD/R. ${ }^{*} \mathrm{P}<0.05,{ }^{* *} \mathrm{P}<0.01,{ }^{* * *} \mathrm{P}<0.001$. OGD/R, oxygen glucose deprivation-reoxygenation; M, model; C, control; TUDCA, tauroursodeoxycholic acid.

showed that early apoptotic cells were $\mathrm{PI}^{-} / \mathrm{Annnexin} \mathrm{V}^{+}$, and late apoptotic cells were $\mathrm{PI}^{+} /$Annexin $\mathrm{V}^{+}$. Most of the cells in the control group were surviving cells. However, following OGD/R treatment, the number of surviving cells decreased remarkably, and more apoptotic cells were detected. As shown in Fig. 3B, the percentage of SH-SY5Y cells at an early stage of apoptosis increased from $8.10 \pm 1.27$ to $11.60 \pm 1.56 \%$ $(\mathrm{P}<0.01)$, and the percentage at a late stage increased from $12.80 \pm 7.35$ to $29.30 \pm 2.26 \%(\mathrm{P}<0.001)$ in the $\mathrm{OGD} / \mathrm{R}$ treated group when compared with those in the control group. In contrast, pretreatment with $100 \mathrm{nl} / \mathrm{ml} \mathrm{JZQKL}$ suppressed early 
A
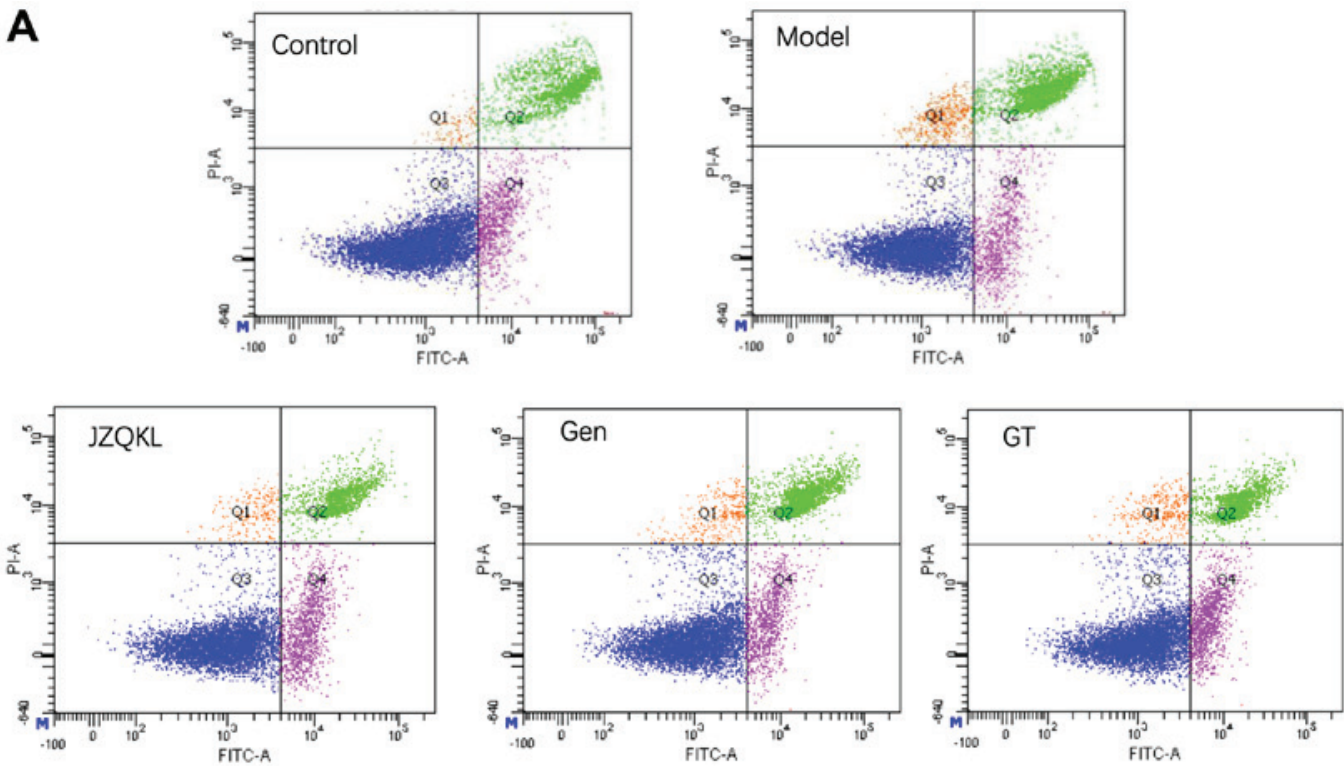

B
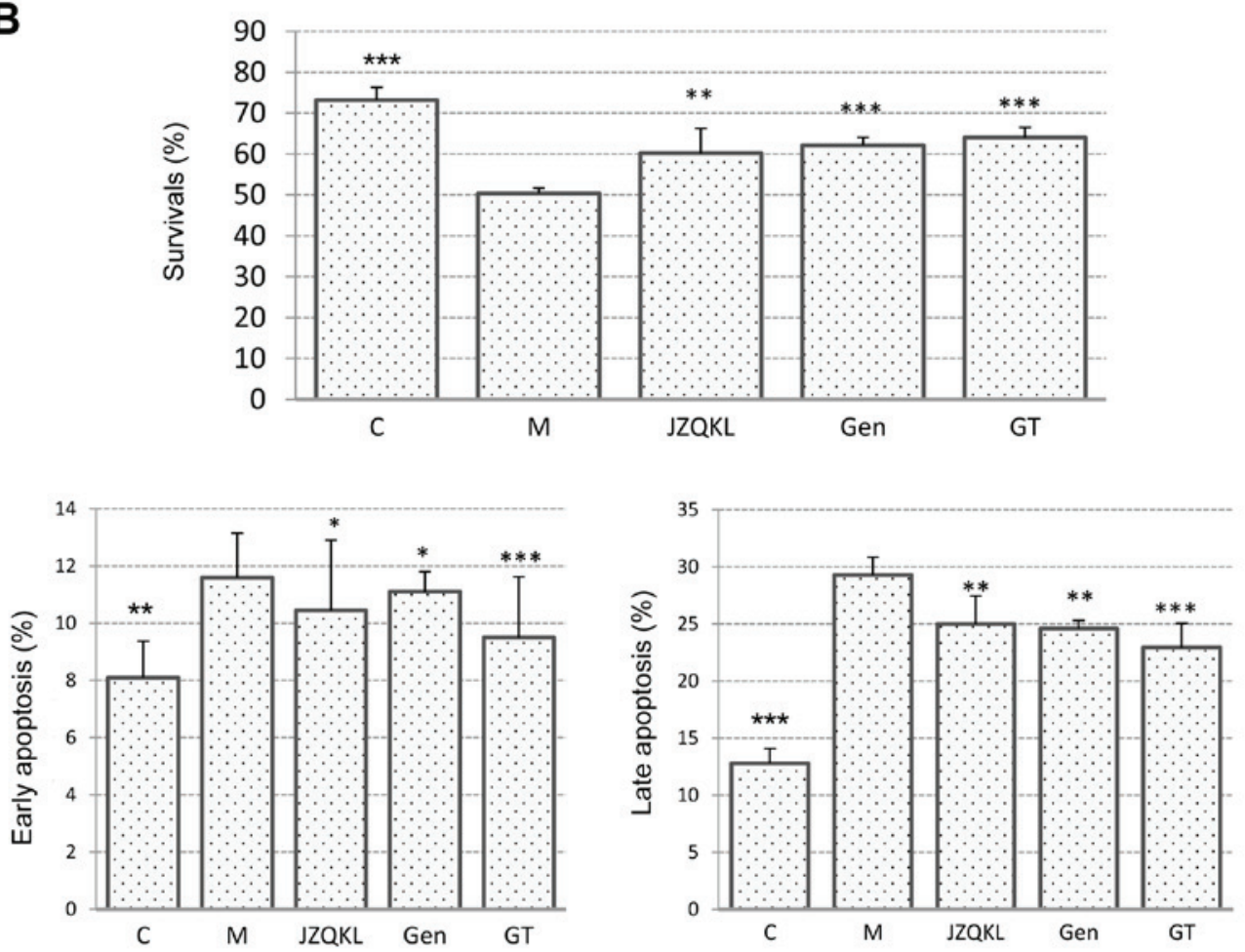

Figure 2. Annexin V-FITC/PI Staining of SH-SY5Y Cells. (A) Representative dot-plots and (B) the percentages of apoptotic cells in SH-SY5Y for the quantitative assessment of cell survival, early stage apoptosis, and late stage apoptosis using Annexin V and PI staining. The cells in the model group (M) and those in the other drug-treated groups were subjected to the OGD/R procedure. Cells cultured under normal conditions served as the control group (C). Data are expressed as means \pm standard deviation of three independent experiments. ${ }^{* *} \mathrm{P}<0.05,{ }^{* * *} \mathrm{P}<0.001$. Gen, Geniposide; M, model; C, control.

and late stage apoptosis rates to $10.45 \pm 2.45 \%(\mathrm{P}<0.05)$ and $25 \pm 9.76 \%(\mathrm{P}<0.01)$, respectively. Pretreatment with $100 \mu \mathrm{g} / \mathrm{ml}$ Geniposide, or a combination of $100 \mu \mathrm{g} / \mathrm{ml}$ Geniposide and $5 \mu \mathrm{g} / \mathrm{ml}$ TUDCA (GT) suppressed the early stage apoptosis rates to $11.1 \pm 0.71 \%(\mathrm{P}<0.05)$ and $9.5 \pm 2.12 \%(\mathrm{P}<0.001)$, respectively. Meanwhile, the percentage of late stage apoptotic cells was reduced to $24.6 \pm 0.85 \%(\mathrm{P}<0.01)$ and $22.95 \pm 0.21 \%$ $(\mathrm{P}<0.001)$, respectively. Thus, the results from the flow cytometry analyses indicate that GT inhibits OGD/R-induced apoptosis better than Geniposide alone although no statistic difference was shown.
Effect of GT on intracellular ROS in SH-SY5Y cells. The levels of ROS were analyzed using flow cytometry by incubating SH-SY5Y cells with the fluorescent dye CM-H2DCFDA (Fig. 3). OGD/R-induced injury significantly increased intracellular ROS levels in SH-SY5Y cells relative to the levels observed in control cells $(\mathrm{P}<0.001)$. The OGD/R-induced increase in ROS was suppressed by GT $(\mathrm{P}<0.01)$. In this experiment $100 \mu \mathrm{M}$ NAC was used as a positive control.

Effect of GT on ER stress and autophagy-related apoptosis. To examine ER stress and autophagy-related protein changes, 

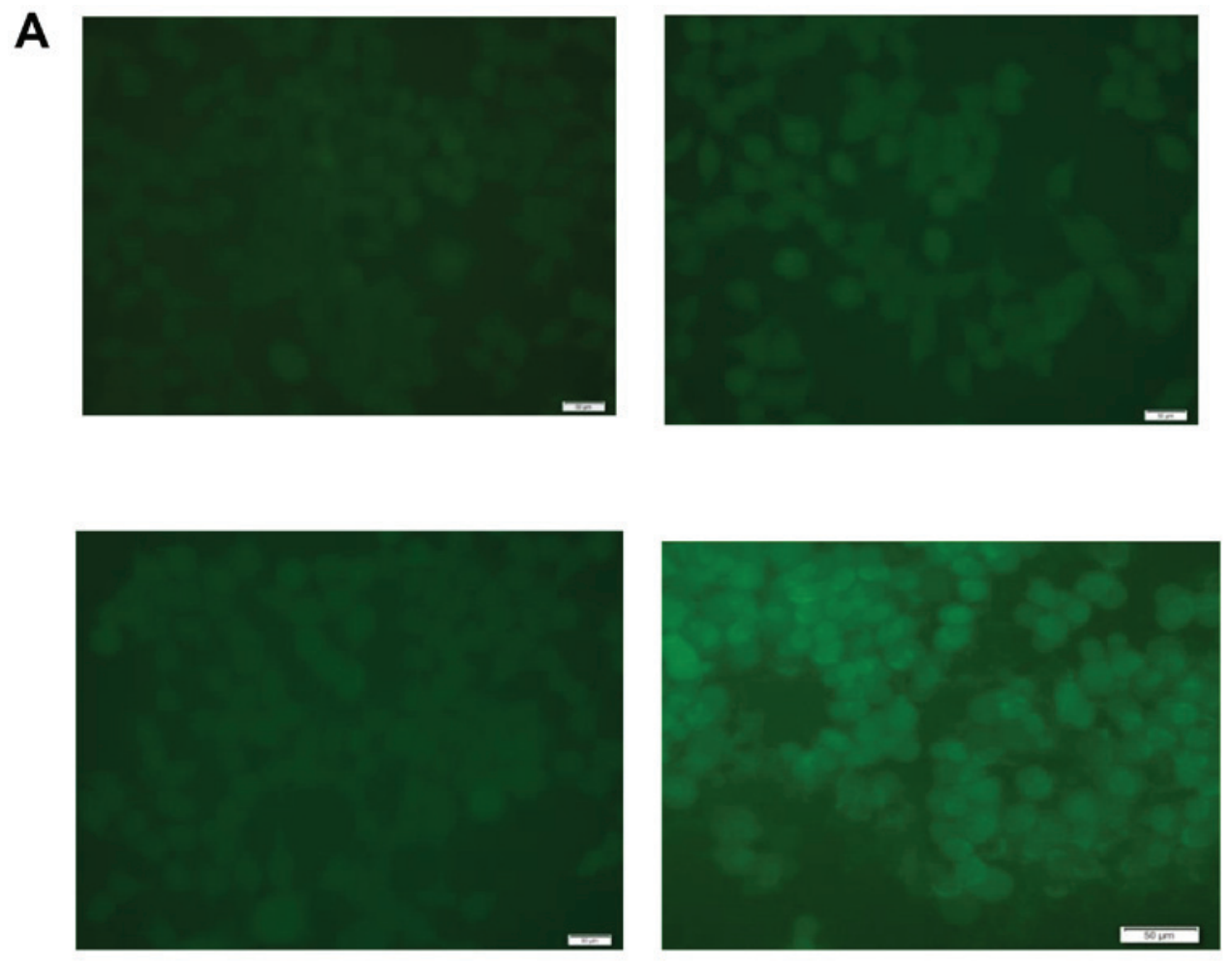

B
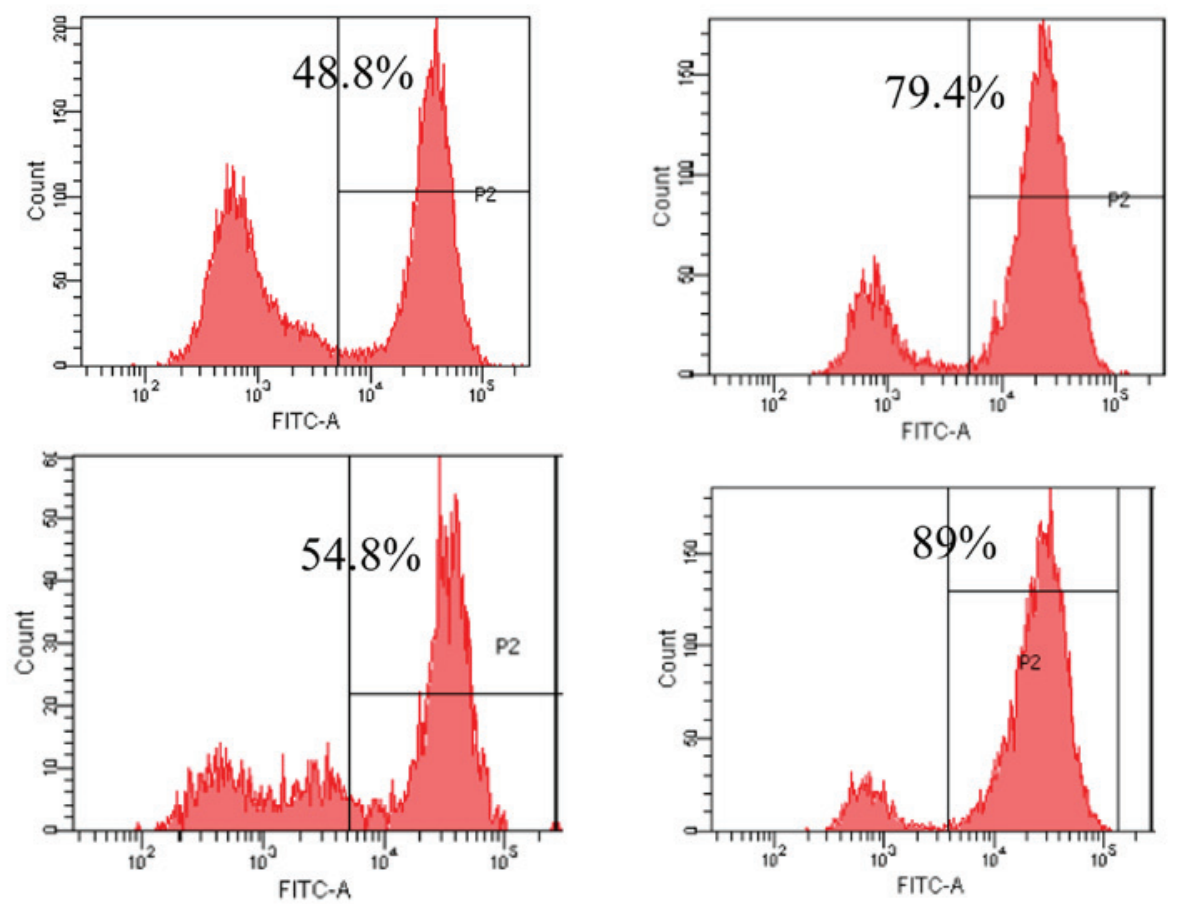

Figure 3. Measurement of ROS levels. (A) Representative fluorescence microscope images of DCFH-DA staining. (B) Percentages of ROS-positive cells measured by flow cytometry. ROS, reactive oxygen species.

western blot and real-time polymerase chain reaction (RT-PCR) analyses were carried out to determine the expression levels of $\mathrm{C} / \mathrm{EBP}$ homology protein (CHOP) and beclin 1 . As shown in Fig. 4, ODG/R altered CHOP and beclin 1 mRNA levels. JZQKL and GT both dramatically reduced OGD/R-induced CHOP $(\mathrm{P}<0.05, \mathrm{P}<0.05)$ and beclin $1(\mathrm{P}<0.01, \mathrm{P}<0.001)$ mRNA levels. The protein levels of $\mathrm{CHOP}(\mathrm{P}<0.001)$ and beclin $1(\mathrm{P}<0.001)$ were reduced in the presence of GT. These results suggest a mechanism by which combined treatment with Geniposide and TUDCA might inhibit OGD/R-induced changes in $\mathrm{CHOP}$ and beclin 1 levels.

\section{Discussion}

Pharmacodynamic constituents from natural medicines have been investigated for the treatment of ischemic stroke. 
A
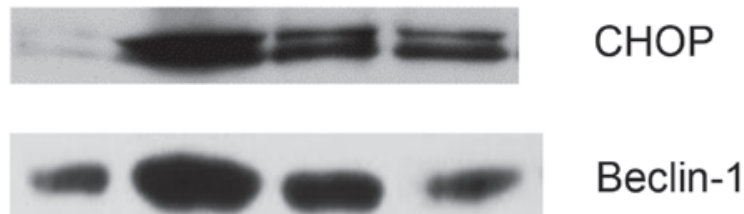

$\beta$-actin
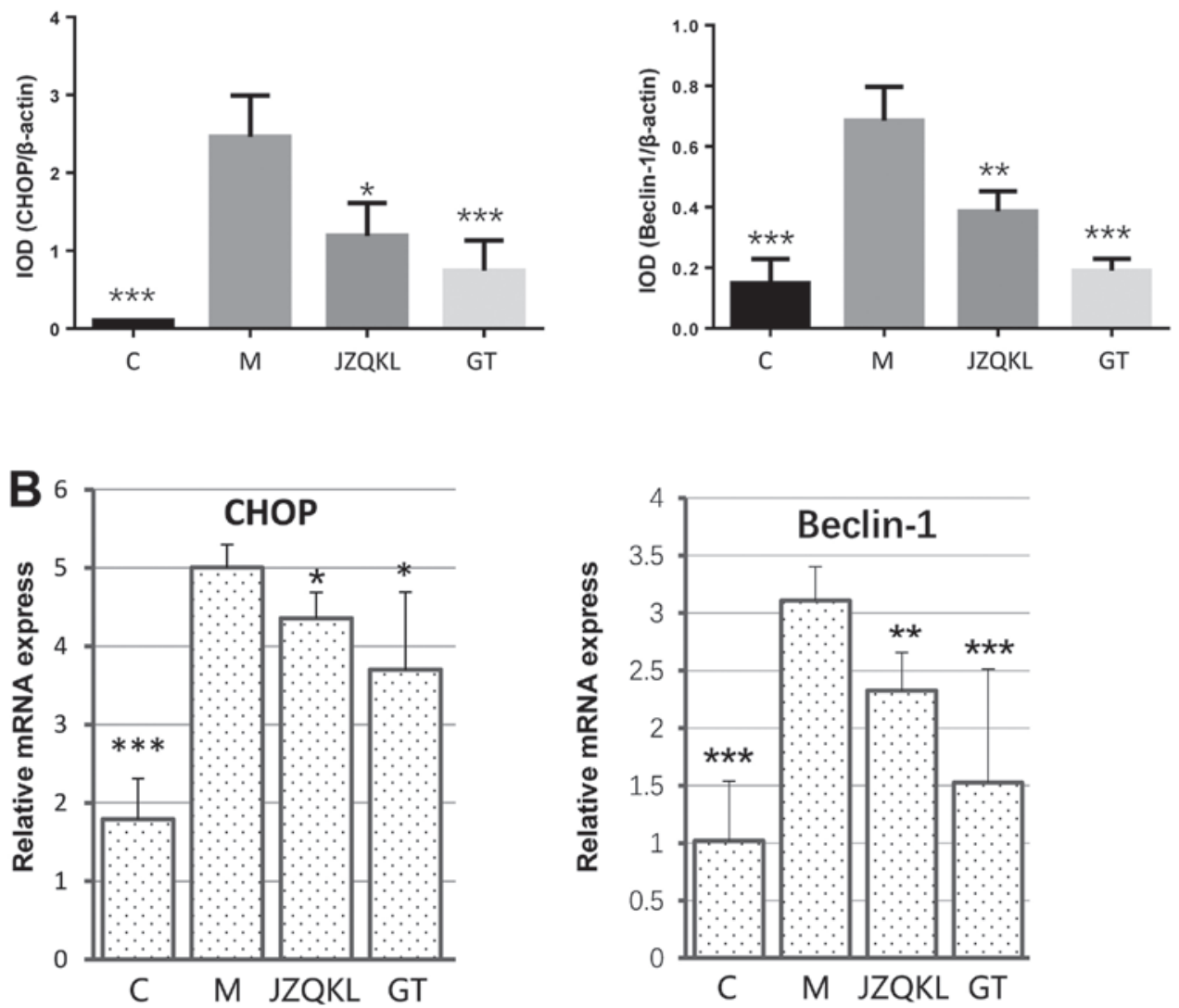

Figure 4. CHOP and beclin 1 protein and mRNA levels. (A) Western blot analysis of CHOP and beclin 1 protein levels, with $\beta$-actin used as a protein loading control. The cells in the model group (M), and those in the JZQKL and Geniposide + TUDCA-treated groups were subjected to the OGD/R procedure. The cells were cultured under normal conditions in the control group (C). (B) RT-PCR analysis of CHOP and beclin 1 mRNA levels. Data are expressed as means \pm standard deviation $(\mathrm{n}=3) .{ }^{*} \mathrm{P}<0.05,{ }^{* *} \mathrm{P}<0.01,{ }^{* * *} \mathrm{P}<0.001$. JZQKL, Jingzhi Qingkailing; GT, Geniposide + TUDCA; CHOP, C/EBP homology protein; M, model; C, control.

Multi-component treatments, characterized by the combination of two or more agents that interact with multiple targets simultaneously, are considered to be rational and efficient forms of therapy for the treatment of complex diseases (19). According to the results obtained from the CCK-8 and LDH assays, proteins secreted from OGD/R-activated SH-SY5Y cells are involved in the neuronal cell damage observed following treatment, indicating that $\mathrm{OGD} / \mathrm{R}$ induces neurotoxicity in SH-SY5Y cells. Here, Geniposide had neuroprotective effects at all concentrations examined, while TUDCA was found to have neuroprotective effects at $25 \mu \mathrm{g} / \mathrm{ml}$ only. Other groups have found geniposide inhibits H/R-induced myocardial apoptosis by reversing mitochondrial dysfunction (20), protects rat insulinoma cells from apoptosis in high-glucose concentrations (21) and attenuate $\mathrm{A} \beta$-induced and rotenone-induced neuronal injury by inhibiting mitochondrial dysfunction and oxidative stress $(9,22)$.
Interestingly, a synergistic protective effect of Geniposide and TUDCA (GT) was observed following OGD/R-induced SH-SY5Y cell injury, especially at a constant 20:1 Geniposide:TUDCA ratio. However, GT had no effect on LDH leakage, suggesting that GT improved SH-SY5Y cell survival through pathways other than those involving LDH.

Apoptosis occurs in many multicellular organisms and arises as part of the normal physiological process in the body (23). Our data show that GT inhibited both early and late stage apoptosis, and was more effective than JZQKL or Geniposide alone. Oxidative stress during neuronal I/R occurs because of the excessive generation or accumulation of free radicals or their oxidation products (24). Excessive ROS destroys mitochondrial membrane integrity, leading to cytochrome $\mathrm{c}$ and apoptosis-inducing factor (AIF) release, caspase activation, and ultimately apoptosis (25). Our data showed that combined treatment with Geniposide and TUDCA decreases 
OGD/R-induced ROS levels, which means ROS may be a potential target for this combination.

The ER performs several functions, including protein folding and transport, and regulation of intracellular calcium concentrations (26). Cells trigger the unfolded protein response (UPR) as a self-protective mechanism upon disruption of ER functions via the accumulation of unfolded/misfolded proteins in the ER. ER stress-mediated apoptosis is triggered by the induction of CHOP (27). In the present study, GT induced the protein and gene expression of CHOP better than JZQKL, suggesting an anti-ER stress-related role in conditions of OGD/R. Autophagy is a cellular defense mechanism that involves the degradation and recycling of cytoplasmic constituents (28). Beclin 1 is an important marker of the stage of the autophagy process (29). Our data show that GT induces the protein and gene expression of beclin 1, suggesting a potential anti-autophagy effect of GT. In conclusion, our results showcase the potential of the combined use of Geniposide and TUDCA to modulate the toxic effects resulting from OGD/R injury. GT treatment may have potential anti-apoptotic, anti-oxidative, anti-ER stress, and anti-autophagy effects. Thus, the use of a multi-targeted treatment approach may be beneficial for the treatment of complex diseases.

\section{Acknowledgements}

The present study was supported by the National Natural Science Foundation of China (81430102, 81373886 and 81303260) and the Classical Prescription Basic Research Team of the Beijing University of Chinese Medicine. The authors thank Elsevier Language Services for providing language assistance and for proofreading the manuscript.

\section{References}

1. Fan YY, Hu WW, Nan F and Chen Z: Postconditioning-induced neuroprotection, mechanisms and applications in cerebral ischemia. Neurochem Int 107: 43-56, 2017.

2. Portt L, Norman G, Clapp C, Greenwood M and Greenwood MT: Anti-apoptosis and cell survival: A review. Biochim Biophys Acta 1813: 238-259, 2011.

3. Kong FJ, Wu JH, Sun SY and Zhou JQ: The endoplasmic reticulum stress/autophagy pathway is involved in cholesterol-induced pancreatic $\beta$-cell injury. Sci Rep 7: 44746, 2017.

4. Cheng F, Zhong X, Lu Y, Wang X, Song W, Guo S, Wang X, Liu D and Wang Q: Refined qingkailing protects MCAO mice from endoplasmic reticulum stress-induced apoptosis with a broad time window. Evid Based Complement Alternat Med 2012: $567872,2012$.

5. Xu XL, Ma CY, Wang XQ, Wang GL, Zhai CM, Yue WC, Li CX, Zhang XY, Shen XD, Mu J, et al: Comparative study of cholic acid compounds of bezoar on anti-cerebral infarction and regulating endoplasmic reticulum stress. Drug Evaluation Res 40: 11-19, 2017 (In Chinese).

6. Cao K and Tong CW: Research progress of pharmacological effects and application of pine needle. J Mianyang Norm Univ, 2015 (In Chinese).

7. Li C, Pan Y and Jia X: Effects of Huangqin (dried root of scutellaria baicalensis) and Zhizi (dried fruit of Gardenia jasminoides) used in combination on ischemic cascade reaction in the rat model of focal cerebral ischemia and reperfusion. J Beijing Univ Tradit Chin Med, 2002.

8. Liu J, Yin F, Zheng X, Jing J and Hu Y: Geniposide, a novel agonist for GLP-1 receptor, prevents $\mathrm{PC} 12$ cells from oxidative damage via MAP kinase pathway. Neurochem Int 51: 361-369, 2007.

9. Zhao C, Lv C, Li H, Du S, Liu X, Li Z, Xin W and Zhang W: Geniposide protects primary cortical neurons against oligomeric A 1 -42-induced neurotoxicity through a mitochondrial pathway. PLoS One 11: e0152551, 2016.
10. Lv C, Wang L, Liu X, Yan S, Yan SS, Wang Y and Zhang W: Multi-faced neuroprotective effects of geniposide depending on the RAGE-mediated signaling in an Alzheimer mouse model. Neuropharmacology 89: 175-184, 2015.

11. Keene CD, Rodrigues CM, Eich T, Chhabra MS, Steer CJ and Low WC: Tauroursodeoxycholic acid, a bile acid, is neuroprotective in a transgenic animal model of Huntington's disease. Proc Natl Acad Sci USA 99: 10671-10676, 2002.

12. Rodrigues CM, Sola S, Nan Z, Castro RE, Ribeiro PS, Low WC and Steer CJ: Tauroursodeoxycholic acid reduces apoptosis and protects against neurological injury after acute hemorrhagic stroke in rats. Proc Natl Acad Sci USA 100: 6087-6092, 2003.

13. Fernández-Sánchez L, Lax P, Pinilla I, Martín-Nieto J and Cuenca N: Tauroursodeoxycholic acid prevents retinal degeneration in transgenic $\mathrm{P} 23 \mathrm{H}$ rats. Invest Ophthalmol Vis Sci 52: 4998-5008, 2011.

14. Rodrigues CM, Spellman SR, Solá S, Grande AW, Linehan-Stieers C, Low WC and Steer CJ: Neuroprotection by a bile acid in an acute stroke model in the rat. J Cereb Blood Flow Metab 22: 463-471, 2002.

15. Amaral JD, Viana RJ, Ramalho RM, Steer CJ and Rodrigues CM: Bile acids: Regulation of apoptosis by ursodeoxycholic acid. J Lipid Res 50: 1721-1734, 2009.

16. Gaspar JM, Martins A, Cruz R, Rodrigues CM, Ambrósio AF and Santiago AR: Tauroursodeoxycholic acid protects retinal neural cells from cell death induced by prolonged exposure to elevated glucose. Neuroscience 253: 380-388, 2013.

17. Zhong XM, Ren XC, Lou YL, Chen MJ, Li GZ, Gong XY and Huang Z: Effects of in-vitro cultured calculus bovis on learning and memory impairments of hyperlipemia vascular dementia rats. J Ethnopharmacol 192: 390-397, 2016.

18. Fu WJ, Lei T, Yin Z, Pan JH, Chai YS, Xu XY, Yan YX, Wang ZH, $\mathrm{Ke} \mathrm{J}, \mathrm{Wu} \mathrm{G}$, et al: Anti-atherosclerosis and cardio-protective effects of the Angong Niuhuang Pill on a high fat and vitamin D3 induced rodent model of atherosclerosis. J Ethnopharmacol 195: 118-126, 2017.

19. Wang J, Hou J, Lei H, Fu J, Pan Y and Liu J: Synergistic neuroprotective effect of microglial-conditioned media treated with geniposide and ginsenoside Rg1 on hypoxia injured neurons. Mol Med Rep 12: 5328-5334, 2015.

20. Jiang YQ, Chang GL, Wang Y, Zhang DY, Cao L and Liu J: Geniposide prevents hypoxia/reoxygenation-induced apoptosis in $\mathrm{H} 9 \mathrm{c} 2$ cells: Improvement of mitochondrial dysfunction and activation of GLP-1R and the PI3K/AKT signaling pathway. Cell Physiol Biochem 39: 407-421, 2016.

21. Guo LX, Liu JH, Zheng XX, Yin ZY, Kosaraju J and Tam KY: Geniposide improves insulin production and reduces apoptosis in high glucose-induced glucotoxic insulinoma cells. Eur J Pharm Sci: pii: S0928-0987(17)30173-2, 2017.

22. Li L, Zhao J, Liu K, Li GL, Han YQ and Liu YZ: Geniposide prevents rotenone-induced apoptosis in primary cultured neurons. Neural Regen Res 10: 1617-1621, 2015.

23. Gordeziani M, Adamia G, Khatisashvili G and Gigolashvili G: Programmed cell self-liquidation (apoptosis). Ann Agrarian Sci 15: 148-154, 2017.

24. Narne P, Pandey V and Phanithi PB: Interplay between mitochondrial metabolism and oxidative stress in ischemic stroke: An epigenetic connection. Mol Cell Neurosci 82: 176-194, 2017.

25. Wu TS, Liao YC, Yu FY, Chang CH and Liu BH: Mechanism of patulin-induced apoptosis in human leukemia cells (HL-60). Toxicol Lett 183: 105-111, 2008.

26. Ogen-Shtern N, Ben David T and Lederkremer GZ: Protein aggregation and ER stress. Brain Res 1648: 658-666, 2016.

27. Senft D and Ronai ZA: UPR, autophagy, and mitochondria crosstalk underlies the ER stress response. Trends Biochem Sci 40: 141-148, 2015.

28. Towers CG and Thorburn A: Therapeutic targeting of autophagy. EBioMedicine 14: 15-23, 2016.

29. Maejima Y, Isobe M and Sadoshima J: Regulation of autophagy by beclin 1 in the heart. J Mol Cell Cardiol 95: 19-25, 2016.

c) (i) $\Theta$ This work is licensed under a Creative Commons Attribution-NonCommercial-NoDerivatives 4.0 International (CC BY-NC-ND 4.0) License. 